\title{
Über den Einfluß der Reizung des Gehirnes (Oblongata) mittels Wechselströme auf das herzhemmende und vaso- motorische Zentrum sowie auf die Atmung ${ }^{1}$ ).
}

\author{
Von \\ Prof. Dr. G. Bikeles $\uparrow$ und Dr. L. Zbyszewski.
}

(Aus dem physiologischen Institut der Universität in Lemberg [Vorstand Hofrat Prof. Dr. A. Beck].)

Mit 4 Textabbildungen.

(Eingegangen am 15. August 1918.)

Die Verwendung von Wecbselströmen als Hirnreizung, und zwar ohne vorausgegangene blutige Operation, nahm zuerst Prévost vor.

Pr évost wie seine Schüler beschäftigte dabei einzig die Frage einer experimentell zu erzeugenden Epilepsie beim Tier, und Pr évost meinte durch eine Hirnreizung mittels Wechselströme bei Anlegung einer Elektrode an den harten Gaumen, der anderen Elektrode an den Nacken, beim Hund und Kaninchen eizen typischen, mit dem klinischen Bilde beim Menschen vollständig identischen epileptischen Anfall erzeugen zu können.

Einer von uns [Bikeles²)] führte zahlreiche Hirnreizungen mittels Wechselströmen beim Hund und Kaninchen, ganz naciı dem Vorgehen von Prévost aus, konnte aber die Äußerung von Prévost über die vollständige Identität des in derartigen Versuchen erzeugten Anfalls mit dem bekannten Bild des epileptischen Anfalles nicht bestätigen.

Im Beginn der Reizung stellt sich wohl ein höchstgradiger Strecktonus des Rumpfes und der Extremitäten ein, derselbe entfaltet sich jedoch - abweichend vom momentanen und brüsken Auftreten des tonischen Stadiums des epileptischen Anfalls beim Menschen - sukzessive und ziemlich langsam.

Bei länger dauernder Reizung hört der tonische Krampf schon im Laufe der Raizung auf, und zwar ganz allmählich. Hierauf stellén sich meist klonische Krämpfe ein, die aber - wiederum im Gegensatz zur klassischen Epilepsie - nicbt unmittelbar dem tonischen Stadium folgen, sondern erst nach geringeren oder größeren Pausen; manchmal traten die klonischen Krämpffe sogar erst nach Einstellung der Reizung ein.

Kann man aber auch die Verwendung von Wechselströmen als

1) Diese wie die folgende Arbeit gingen der Redaktion bereits am 15. VIII. $1918 \mathrm{zu}$, jedoch fehlten die Figuren und einige Aufklärungen über den Text. Durch die Revolution war dann lange die Verbindung unterbrochen, Briefe und Klischees gingen später verloren, so daß von den Autoren lange keine Nachrichten einliefen. Auf eine Anfrage teilte uns schließlich Prof. Beck mit, daß Prof. Bikeles einer mörderischen Kugel bei einem Straßenkampf zum Opfer gefallen ist. Da die Figuren erst vor kurzem eintrafen, hat sich die Veröffentlichung bedauerlicherweise so. lange verzögert. 'April 1920.

2) Neurol. Zentralbl. 1916, Nr. 8. 


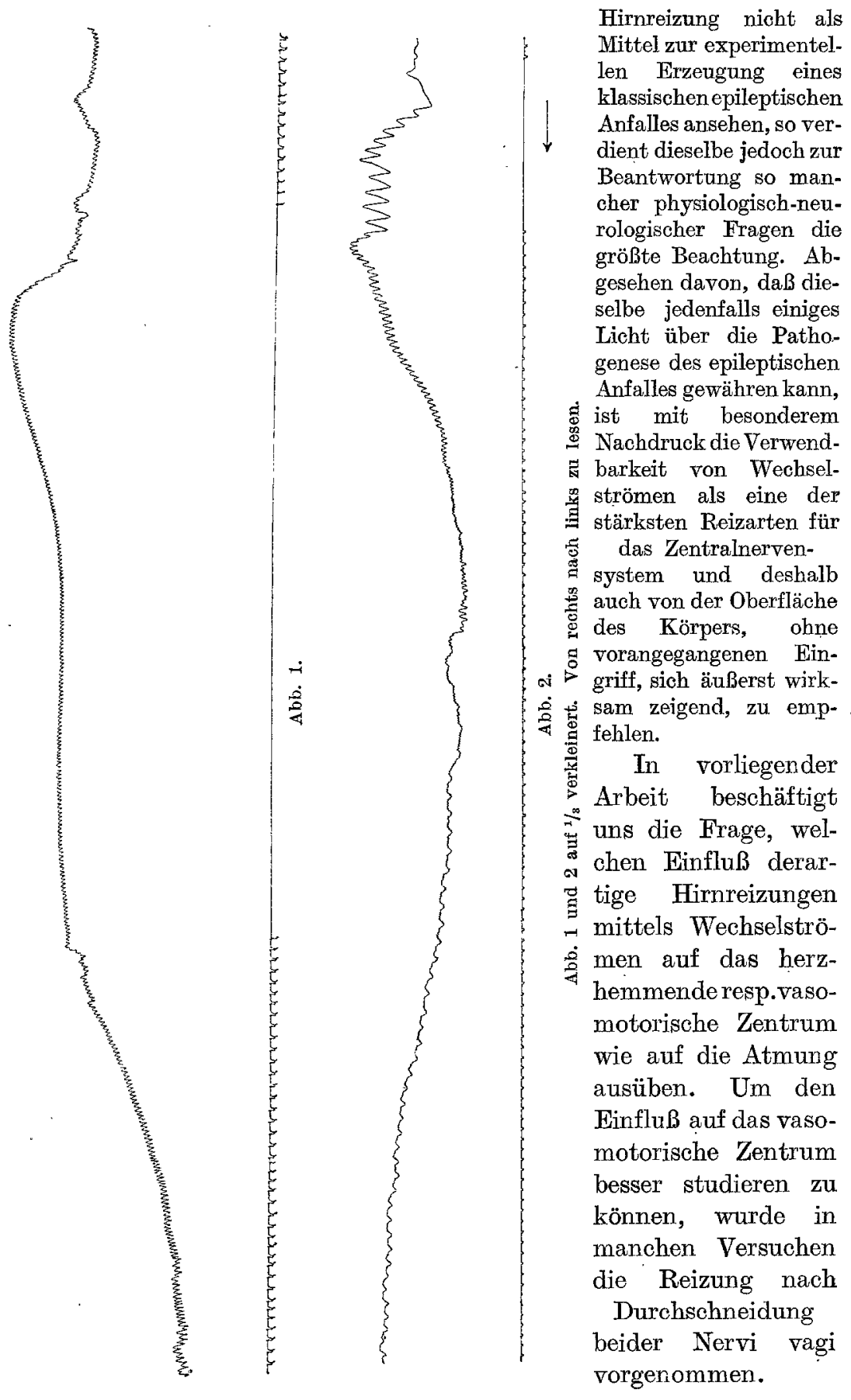


Als Quelle für die angewandten Wechselströme benützten wir die Zuleitung. der elektrischen Stadtzentrale. Zwischen Tier und Elektrizitätsquelle war ein Stöpselrheostat und ein Weston-Milliampèremeter für Wechselströme, eingerichtet zur Messung von Intensitäten bis 75 MA., eingeschaltet.

Bei allen diesen Versuchen befand sich die eine Elektrode am Nacken, die andere (knopfartige schmälere) am Gaumen.

Der Blutdruck wurde mittels des Ludwigschen Kymographion registriert.

Die Atmung wurde nach stattgehabter Tracheotomie vermittels Flaschenvorrichtung und Mareyscher Kapsel registriert.

Zur Verwendung kamen Hunde und Kaninchen ${ }^{\mathbf{1}}$ ).

Die erhaltenen Resultate veranschaulichen, was den Kreislauf anbelangt die beigefügte Tabelle, betreffend die Atmung aber, die zusammengestellten Versuchsprotokolle. (S. 160.)

Úber den Einfluß auf den Kreislauf beansprucht in der Tabelle vor allem die Rubrik ,anfängliche vollständige Hemmung“ eine nähere Auseinandersetzung.

Vor dem Erscheinen gewöhnlicher typischer Vaguspulse kam ïber a us häufig, sowohl bei Hunden wie bei Kaninchen, bei starken und mittelstarken Strömen - bei individueller Verschiedenheit - eine allmählich abfallende Kurve ohne jede Andeutung von Pulsen zum Voŕschein a!s Ausdruck einer länger andauernden Hemmung der Herztätigkeit (Abb. I und 2).

Nur bei einem Hund (Tab., Versuch 4) und bei einem Kaninchen (Tab., Ver'such 2) blieb diese Hemmung bei allen Reizungen aus, und es erfolgte jedesmal alsbaldige Blutdrucksteigerung mit gewöhnlichen Vaguspulsen. Manchmal ging dieser Hemmung eine sehr kurz dauernde Spur einer Blutdrucksteigerung voraus. Immer aber war die Hemmung eine anfängliche, d. h. dieselbe erfolgte niemals in der Mitte oder gegen Ende der Reizung, sondern in Beginn derselben, und wenn die Reizung etwas länger andauerte, wie es bei unseren Versuchen fast immer der Fall war, hörte dieselbe noch im Laufe der Reizung auf.

Vorgenommene Messungen sowohl der Zeitdauer dieser Hemmung als auch des dabei erfolgten Absinkens des Blutdruckes stellen sich folgendermaßen dar:

Vers. I. Reizung 75 MA. . . . . . . 9

Hund:

Dauer d. Hemmung in Sekunden

Reizung $1000 \mathrm{Ohm} . . . .12$

Vers. II. Reizung 65 MA. . . . . . . 2,5

Vers. III. Reizung 60 MA. . . . . . 21

Vers. IV. Reizung 65 MA. . . . . . . 8

Reizung $1000 \mathrm{Ohm}$. . . . 8

$\begin{array}{cc}\begin{array}{c}\text { Abfall des } \\ \text { von } \mathrm{mm} \mathrm{Hg}\end{array} & \begin{array}{c}\text { Blutdruckes } \\ \text { auf } \mathrm{mm} \text { Hg }\end{array} \\ 196 & 136 \\ 150 & 96 \\ 156 & 90 \\ 130 & 22 \\ 204 & 116 \\ 186 & 116\end{array}$

1) Wegen ungewöhnlicher Verhältnisse infolge der Nähe des Kriegsschauplatzes in den Jahren 1916/17 war die Beschaffung der Versuchstiere, besonders von Hunden mit großen Schwierigkeiten verbunden. Die Arbeit wurde abgeschlossen Ende des Jahres 1917 und wurde im März 1918 an die Redaktion abgesandt, kam aber (wie wir im 'Juni 1918 erfuhren) in Verlust. 
TabelieI. Einfuß von Hirnreizung (Oblongata) mittels Wechselströme auf den Kreis lauf. 1. Hund.

\begin{tabular}{|c|c|c|c|c|c|c|c|c|c|}
\hline 㞼 & 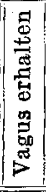 & 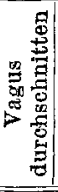 & $\begin{array}{l}\text { Reiz- } \\
\text { dauer } \\
\text { in } \\
\text { Sek. }\end{array}$ & $\begin{array}{l}\text { Milli- } \\
\text { ampère }\end{array}$ & $\begin{array}{l}\text { A n fängliche } \\
\text { vollständige } \\
\text { Hemmung } \\
\text { der Herzaktion } \\
\text { (BIutdruck- } \\
\text { senkung) }\end{array}$ & $\begin{array}{l}\text { S o f ortige } \\
\text { Blutdruck- } \\
\text { steigerung }\end{array}$ & $\begin{array}{l}\text { Nachträgliche } \\
\text { Vaguspulse }\end{array}$ & $\begin{array}{l}\text { Nachträgliches } \\
\text { tiefes Sinken } \\
\text { d. Blutdruckes }\end{array}$ & Anmerkung \\
\hline 1 & + & - & $30-60$ & $\begin{array}{l}72-75 \text { u. } \\
\text { darüber }\end{array}$ & $\stackrel{+}{+}$ & - & + & - & \\
\hline $2 a$ & + & - & 60 . & $\begin{array}{l}1200 \text { Ohm } \\
\text { (über } 75 \text { ) }\end{array}$ & 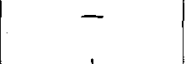 & $\stackrel{+}{+}$ & - & - & $\begin{array}{l}\text { Eine Reizung, ebenfalls } \\
\text { b.1200 Ohm, verursacht }\end{array}$ \\
\hline $2 b$ & + & - & 60 & 65 & $(1$ Reizung $)$ & - & + & - & $\begin{array}{l}\text { bei geringfügigen Herz- } \\
\text { kontraktionen soforti- }\end{array}$ \\
\hline 3 & + & -- & 60 & 60 & (1 Reizung) & . $\quad-$ & + & - & $\begin{array}{l}\text { ge sehwache Steige- } \\
\text { rung des Blutdruckes. }\end{array}$ \\
\hline 4 & + & - & 60 & $40-75$ & - & $\begin{array}{c}+ \\
(6 \text { Reizung. })\end{array}$ & + & - & $\begin{array}{l}\text { Bei } 5 \text { Reizungen bedeu- } \\
\text { tende Steigerung, bei } \\
\text { der letzten schwache. }\end{array}$ \\
\hline $5 a$ & + & - & 30 & $\begin{array}{c}\text { weniger als } \\
5-25\end{array}$ & 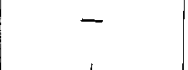 & $\frac{+}{\text { (9 Reizung.) }}$ & - & $\rightarrow$ & $\begin{array}{l}\text { Eine Reizung bei } 33 \mathrm{MA} \text {. } \\
\text { ähnlich wie die in Be- }\end{array}$ \\
\hline $5 b$ & & - & 30 & 46 und 60 & (2 Reizungen) & - & + & - & merkung, Versuch 1,2 . \\
\hline $6 a$ & + & - & $15-\dot{2} 5$ & 50 & - & $\begin{array}{c}+ \\
\text { (1 Reizung) }\end{array}$ & & - & $\begin{array}{l}\text { *) In beiden Versuchen } \\
\text { wurden die Nn. vagi }\end{array}$ \\
\hline $6 \mathrm{~b}$ & & 一 & & 65 und 75 & (2 Reizungen) & - & + & 一 & $\begin{array}{l}\text { erst nach vorausgegan- } \\
\text { genen Reizung. durch- }\end{array}$ \\
\hline 7 & - & + & 60 & $\begin{array}{l}45 \text { bis über } \\
75\end{array}$ & - & $\frac{+}{(6 \text { Reizung. })}$ & $\therefore$ & + & schnitten, und die hier \\
\hline 8 & - & + & 60 & 61 & - & (1 Reizung) & - & $+*$ & $\begin{array}{l}\text { te bereits zur Agonie, } \\
\text { daher an sich nicht } \\
\text { überreizend, jedoch }\end{array}$ \\
\hline 9 & - & + & 60 & $7 \tilde{5}$ & - & (1 Reizung) & - & $\left.+*^{*}\right)$ & $\begin{array}{l}\text { Kurve ganz analog wie } \\
\text { bei Versuch } 7 \text {. }\end{array}$ \\
\hline 10 & - & + & 15 & $\begin{array}{c}73-75 \\
\text { u. } 500 \text { Ohm }\end{array}$ & - & $\begin{array}{c}+ \\
\text { (3Reizung.) }\end{array}$ & - & + & $\begin{array}{l}\text { Bei } 73 \text { u. } 75 \text { Senkung, nur } \\
\text { auffallend steil, aber } \\
\text { nicht unter dem Blut- } \\
\text { druck vor der Reizung. }\end{array}$ \\
\hline
\end{tabular}

2. Kaninchen.

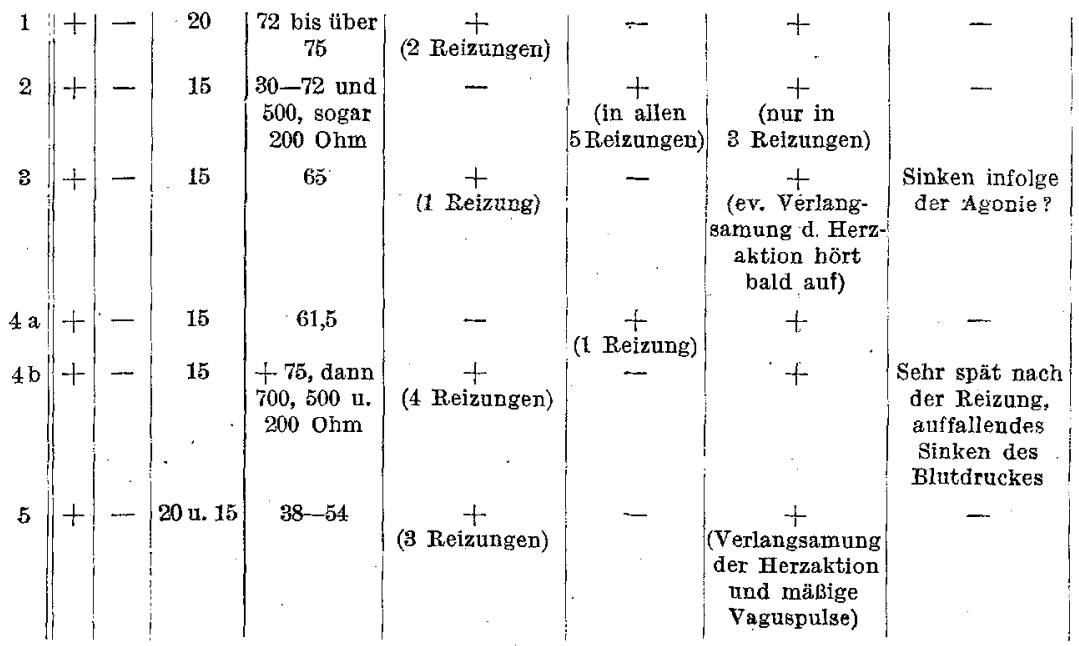




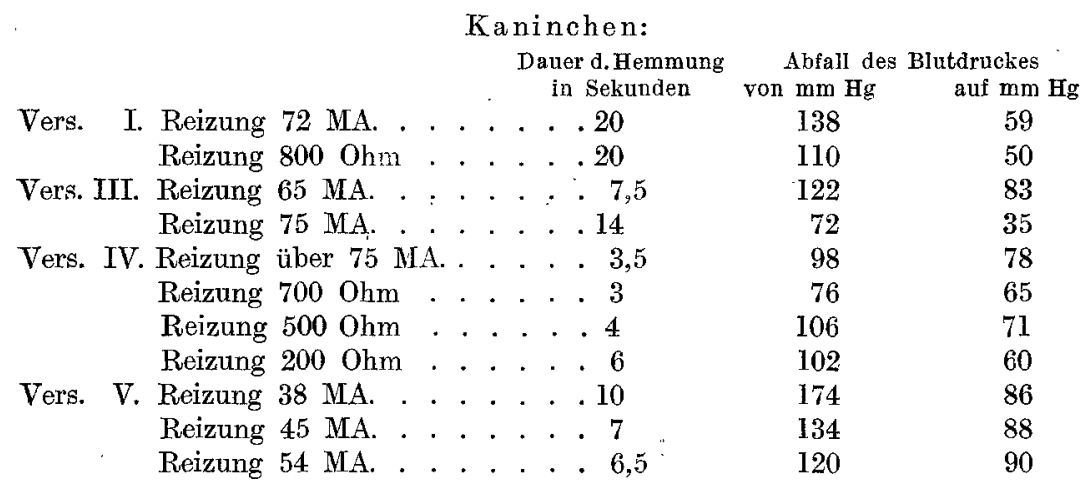

Aus obigem ist ersichtlich, daß die vollständige He m mung nur a usnahmsweise bis zu 20 Sekunden andauert; meistenshält dieselbe bedeutend weniger lang an. Bemerkenswert ist, daß bei manchen Versuchstieren die Dauer der Hemmung nur um ein gerirges schwankt und daß bei diesen Versuchstieren das erfolgte Absinken des Blutdruckes auch bei mehreren Reizungen auf ungefähr gleichem Niveau sich bewegt, obwohl die angewandte Stromintensität eine verschiedene war.

Der Umstand, daß die vollständige Hemmung der Herzaktion bei Reizung mittels Wechselströmen nur von kurzer Dauer ist, wirft zugleich einiges Licht auf das Verhalten des bei diesen Reizungen regelmäßig einsetzenden Extensionstonus, welches letzteres Phänomen ebenfalls - wie bereits erwähnt - im Beginn der Reizung zum Vorschein kommt und noch im Verlaufe der Reizung schwindet. Die Dauer des Stresktonus ist zwar nicht identisch mit der Dauer der Hemmung der Herzalktion, áber beide Erscheinungen als Ausdruck einer starken Reizung der entsprechenden Zentra sind anfängliche, $d$. h. im Beginn der Reizung auftretende und nicht bis Schluß der etwas länger dauernden Reizung anhaltende. Gerade die kurze Zeit, durch die die komplette Hemmung in diesen Versuchen anhält, welche wie bereits erwähnt nur ausnahmsweise 20 bei weitem, überwiegend aber nur wenige 'Sekunden dauerte, spricht dafür, daß diese „anfänglichen " Charakter zeigende Hemmung, keineswegs einer Erschöpfung des peripheren Apparates (wie es bei etwaigen Reizungen des peripheren Vagusstumpfes der Fall ist $\left.{ }^{1}\right)$ ), zuzuschreiben ist; vielmehr ist das Aufhören sowohl der Herzhemmung wie des Extensionstonus im Verlaufe der Reizung dadurch bedingt, das die Reizwirkung auf die Zentra gerade im Beginn am wirksamsten ist.

Um die Wirkung dieser Reizart auf das vaso motorische Zentrum ohne Interferrenz des herzhemmenden Zentrums beobachten zu können, wurden bei vier Hunden die Nn. vagi durchschnitten. Bei einem Hunde (Tabelle, Versuchsprotokoll 7) wurde die Druchschneidung der Nn. vagi von vornherein, d. i. vor den überhaupt stattgehabten Reizungen, vorgenommen.

Bei den hierauf vorgenommenen Reizungen zeigte sich nun fol: gendes Verhalten:

1) Von dem in der Regel ganz frappanten Unterschied überzeugten wir uns durch Ausführung entsprechender Versuche.

Pflügers Archiv f. d. ges. Physiol. Bd. 182. 
Im Beginn der Reizung erfolgte rasch das Maximum der Blutdrucksteigerung, um aber bald im weiteren Verlaufe der Reizung mehr oder weniger, wenn auch mäßig, abzunehmen, während gegen Ende, seltener beim Aufhören der Reizung ein weiteres sehr auffallendes tiefes Absinken des Blutdruckes sich einstellte, wobei das Minimum nach Ablauf von 10 bis etwa 30 Sekunden nach Aufhören der Reizung erreicht wurde (Abb. 3 und 4).

Im speziellen stellt sich das Ergebnis folgendermaßen dar:
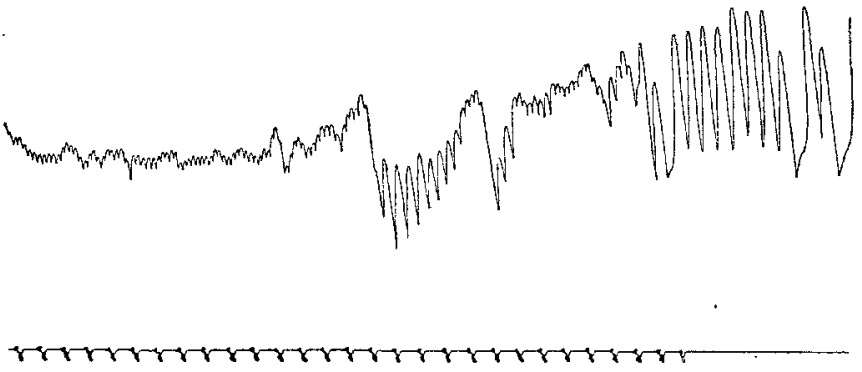

Abb. 4.

$\rightarrow \quad A b b .3$ und 4 auf $1 / 8$ verkleinert. Von rechts nach links zu lesen.

Reizung bei $68 \mathrm{MA}$. von ${ }_{238}^{244} \mathrm{~mm} \mathrm{Hg}$ auf ${ }_{70}^{80} \mathrm{~mm} \mathrm{Hg} \quad$ [erreicht nach 20 Sekunden nach Aufhören der Reizung]. Reizung bei $66 \mathrm{MA}$. Sinken von $\begin{aligned} & 216 \\ & 204\end{aligned} \mathrm{~mm} \mathrm{Hg}$ auf 130 $\mathrm{mm} \mathrm{Hg}$ [nach 10 Sekunden nach Aufhören der Reizung]. Reizung bei 71 MA. Sinken von $\begin{aligned} & 216 \\ & 196\end{aligned} \mathrm{~mm} . \mathrm{Hg}$ a uf 50 (46) $\mathrm{mm} \mathrm{Hg}$ [nach 25 (35) Sekunden nach Aufhören. der Reizung].

Reizung bei $1000 \mathrm{Ohm}$. Sinken von ${ }_{168}^{190} \mathrm{~mm} \mathrm{Hg}$ a uf $56 \mathrm{~mm}$ Hg [nach 32 Sekunden nach Aufhören der Reizung].

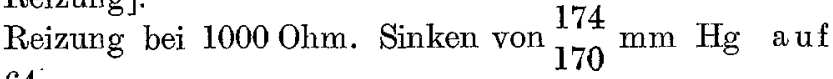
${ }_{56}^{64} \mathrm{~mm} \mathrm{Hg}$ [nach 20 Sekunden nach Aufhören der Reizung]:

Bei den übrigen Hunden wurden Reizversuche zunächst bei intakten Nn. vagi und erst hierauf deren Durchschneidung vorgenommen. Bei einem derselben 
(Tabelle, Versuchsprotokoll 10) wurden drei Reizungen bei durchschnittenen Nn. vagi ausgeführt mit folgendem Ergebnis:

Reizung bei 73 MA. Steiler Abfall von $270 \mathrm{~mm} \mathrm{Hg}$ auf $180 \mathrm{~mm} \mathrm{Hg}$ [in 6 Sekunden nach der Reizung].

Reizung bei $1000 \mathrm{Ohm}$. Steiler Abfall von 276 auf $180 \mathrm{~mm} \mathrm{Hg}$ [in 7 Sekunden nach Aufhören der Reizung].

Reizung bei $500 \mathrm{Ohm}$. Sinken von 264 auf $56 \mathrm{~mm} \mathrm{Hg}$.

Bei zwei Hunden konnte nach Durchschneidung der Nn. vagi nur je eine Reizung vorgenommen werden, da das tiefe Absinken des Blutdruckes (vielleicht auch 'eine Erschöpfung wegen mehrerer vorangegangener Reizungen) die Agonie des Tieres herbeiführte (Tabelle, Versuchsprotokoll 8 und 9).

Ist somit das tiefe Absinken bei den letzten zwei Hunden nicht eindeutig, so scheint uns jedoch die Gestalt der Kurve wie besonders der Umstand, daß vor dieser letzten zur Agonie führenden Reizung der Blutdruck sich nicht wesentlich alteriert zeigte, zur Annahme zu berechtigen, daß der bedeutende Abfall des Blutdruckes nach dieser letzten Reizung weniger auf eine Schwäche der Herzaktion als vielmehr auf eine Lähmung des Vasomotorenzentrums in der Oblongata zurückzuführen ist.

Bei Hunden mit intakten Nn. vagi bot sich uns keine derartige Beobachtung dar. Nur bei einem Kaninchen (Tabelle, Versuchsprotokoll 4) mit erhaltenen Nn. vagi zeigte sich ein ähnliches jedoch nicht ganz identisches Verhalten:

Reizung bei $61,5 \mathrm{MA}$. Sinken von ${ }_{134}^{160} \mathrm{~mm} \mathrm{Hg}$ auf ${ }_{56}^{64} \mathrm{~mm} \mathrm{Hg}$ [in $32 \mathrm{Se}-$ kunden nach Aufhören der Reizung.

Reizung bei über $75 \mathrm{MA}$. Sinken von $\begin{aligned} & 128 \\ & 102\end{aligned} \mathrm{~mm} \mathrm{Hg}$ auf $45 \mathrm{~mm} \mathrm{Hg}$ [in 30 Sekunden. nach Aufhören der Reizung].

Reizung bei über $75 \mathrm{MA}$. Sinken von $124 \mathrm{~mm} \mathrm{Hg}$ auf $40 \mathrm{~mm} \mathrm{Hg}$ [in 27 Sekunden nach Aufhören der Reizung].

Reizung hei $700 \mathrm{Ohm}$. Sinken von $\frac{115}{104} \mathrm{~mm} \mathrm{Hg}$ auf $42 \mathrm{~mm} \mathrm{Hg}$ [in $26 \mathrm{Se}-$ kunden nach Aufhören der Reizung]. Reizung bei 500 Onm. Sinken von $106 \mathrm{~mm} \mathrm{Hg}$ auf $\frac{42}{38} \mathrm{~mm} \mathrm{Hg}$ [in. $25 \mathrm{Se}-$ kunden nach Aufhören der Reizung].

Bei diesem Kaninchen jedoch erfolgte das Absinken des Blutdruckes durchgehend erst nach Aufhören der Reizung, und es zeigte sich manchmal unmittelbar nach Unterbrechung der Reizung noch eine weitere Steigerung des Blutdruckes, der erst dann das Abfallen nachfolgte. $\mathrm{Nach}$ diesem auffallenden Sinken stellte sich allmählich 
jedesmal wiederum ein für dieses Tier normaler Blutdruck ein, von ungefähr über $100 \mathrm{~mm} \mathrm{Hg}$.

Dieses ungemein tiefe Absinken des Blutdruckes (bei Hunden nach durchschnittenen Nn. vagi, bei einem Kaninchen auch bei intakten Nn. vagi) kann nur als vorübergehende komplette Erschöp fung des vasomotorischen Zentrums in der Oblongatain. folge der starken Reizung gedeutet werden. Denn der restierende Blutdruck von über $50 \mathrm{~mm} \mathrm{Hg}$ ist eigentlich speziell, beim Hund, ein solcher, der auch nach Ausschaltung der Oblongata fast verbleibt, als Effekt der vasomotorischen Zentıa des Rückenmarks oder des Rückenmarkstonus für die Vasoconstrictoren ${ }^{1}$ ).

Ein Analogon zu dieser Erschöpfbarkeit des vasomotorischen Zentrums beobachteten wir im Laufe dieser Untersiuchungen auch bezüglich des herzhemmenden Zentrums. In manchen Versuchen nämlich erfolgte beim Hund bei intakten Nn. vagi - nach kompletter Hemmung mit darauffolgenden Vaguspulsen - nicht lange nach Aufhören der Reizung eine hochgradige Beschleunigung der Herzaktion, wobei die Kurve vorübergehend das Bild einer Vaguslähmung zeigte.

Zum vasomotorischen Zentrum zurückkehrend müssen wir nun auf Grund obiger Konstatierungen die gangbare Lehre, welche lautet, da $B$,das vasomotorische Zentrum fast unermüdbar sei", so generell ausgedrückt, als unbegründet betrachten. Höchstens kann man sagen, daß zur Erhaltung des gewöhnlichen Tonus das vasomotorische Oblongatazentrum sich unerschöpfbar zeigt.

1) Nach Abschluß dieser Arbeit führten wir noch zwei ergänzende Versuche an Hunden aus. Zunächst wurde bei denselben Hirn- (Oblongata-) Reizungen bei erhaltenen, nachher bei durchschnittenen $\mathrm{Nn}$. vagi vorgenommen. Bei beiden Hunden verursachte die Reizung bei einer Intensität von 37, 45 und $59 \mathrm{MA}$. keine Erscheinung einer etwaigen Erschöpfung des vasomotorischen Zentrums; stärkere Ströme wandten wir vor der Vagusdurchschneidung nicht an, um nicht das so schwer erhaltbare Tier zu verlieren. Nach Durchschneidung beider Nn. vagi er* folgte bei einem Hund erst bei $500 \mathrm{Ohm}$ (Milliamperemeter ausgeschaltet) ein $\mathrm{Ab}$ sinken von 260 auf $68 \mathrm{~mm} \mathrm{Hg}$ und nach einer Pause mit Wiederansteigen des Blutdruckes, bei abermaliger Reizung bei $500 \mathrm{Ohm}$ erneuerter Abfall von 216 auf $69 \mathrm{~mm}$ $\mathrm{Hg}$; beim zweiten Hund erfolgte nach Durchschneidung beider Nn. vagi sogar erst bei gänzlicher Ausschaltung jedes Widerstandes, ein Absinken von 216 auf $76 \mathrm{~mm} \mathrm{Hg}$ und nach einer Pause mit Wiederzunahme des Blutdruckes auf abermalige Reizung $(\mathrm{Ohm}=\mathrm{Null})$ ein Abfall von 160 auf $60 \mathrm{~mm} \mathrm{Hg}$. Aus obigem folgt also, daß die Erschöpfbarkeit des vasomotorischen Oblongatazentrums beim Hund bedentende individuelle Verschiedenheiten aufweist. Ob die Durchschreidung der Nn. vagi beim Hund irgendeinen fördernden Einfluß auf das Phänomen der Erschöpfloarkeit des vasomotorischen Zentrums hat, läßt sich nicht mit irgendwelcher Bestimmtheit aussagen, da die angewandten Stromintensitäten bei erhaltenen und durchschnittenen $\mathrm{Nn}$. vagi nicht dieselben waren. 
Zusammenstellung des in den einzelnen Versuchen beobachteten Einflusses auf die Atmung bei Reizung mittels

Wechselströme.

A. Kaninchen.

\begin{tabular}{|c|c|c|c|c|c|c|c|}
\hline \multirow{2}{*}{ 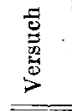 } & \multirow[b]{2}{*}{$\mathrm{Ohm}$} & \multirow{2}{*}{$\begin{array}{l}\text { Milli- } \\
\text { ampère }\end{array}$} & \multirow{2}{*}{$\begin{array}{l}\text { Reiz } \\
\text { dauer } \\
\text { Sek. }\end{array}$} & \multirow{2}{*}{$\begin{array}{l}\text { Verlang- } \\
\text { samung der } \\
\text { Atmung }\end{array}$} & \multicolumn{3}{|c|}{ Hemmung der Atmung } \\
\hline & & & & & anfängliohe & $\begin{array}{c}\text { während der } \\
\text { Reizung }\end{array}$ & $\begin{array}{c}\text { Reizung } \\
\text { uuberdauernd }\end{array}$ \\
\hline \multirow[t]{5}{*}{$\left.I^{ \pm}\right)$} & 12000 & 5 & 30 & & & & \\
\hline & 11000 & $10-12$ & 30 & - & - & + & -.- \\
\hline & 10000 & 13 & 30 & & & $T$ & \\
\hline & 8000 & 15 & 30 & & & & \\
\hline & 6000 & 18 & 30 & 一 & - & - & + \\
\hline \multirow[t]{7}{*}{$\left.\mathrm{II}^{2}\right)$} & $22000\}$ & $\begin{array}{l}\text { ohne Ausschlag } \\
\text { des }\end{array}$ & 30 & $+\eta_{m a ̈ ß i g}$ & $\ldots$ & - & - \\
\hline & $20000\}$ & Ampèremeters & 30 & $+5^{1000018}$ & & & \\
\hline & $\begin{array}{l}18000 \\
16000\}\end{array}$ & 2 & 30 & - & $+\left\{\begin{array}{c}\text { erste } \\
\text { Halfte }\end{array}\right.$ & - & - \\
\hline & 14000 & $2-4$ & 30 & ) & & & \\
\hline & 12000 & $7-8$ & 30 & 一 & - & + & - \\
\hline & 10000 & $10-11$ & 30 & J & & & \\
\hline & 8000 & 13 & 30 & 一 & -- & -- & $\frac{1}{5}$ \\
\hline \multirow[t]{10}{*}{$\left.\mathrm{III}^{3}\right)$} & 22000 & ohne & 30 & \} & $+\int$ kurz- & $\ldots$ & - \\
\hline & $20000\}$ & Ausschlag & 30 & & ${ }^{\top}$ danernd & & \\
\hline & 17000 & 5 & 30 & + seicht & - & 一 & . - \\
\hline & 15000 & $6-7$ & 30 & - & + & 一 & - \\
\hline & 12000 & $8-9$ & 30 & & & & \\
\hline & 10000 & $9-10$ & 30 & . & & & \\
\hline & 8000 & 14 & 30 & - & - & + & - \\
\hline & 6000 & 16 & 30 & & & & \\
\hline & 6000 & 16 & 30 & & & & \\
\hline & 4000 & 23 & 30 & - & - & - & + \\
\hline
\end{tabular}

1) Bei 5,13 und mehr bei 10-12 MA. finden sich Spuren einer sehr seichten und seltenen Atmung während der Hemmungsdauer. Nach Wiederkehr der Atmung ist dieselbe von gewöhnlichem Typus.

2) Verlangsamung der Atmung bei 22,000 und 20000 Ohm betraf die erste Häfte der Reizdauer. Nach Hemmung bei 18000 und $16000 \mathrm{Ohm}$ verlangsamte Atmung, die Reizung sogar überdauernd; während gerade bei den stärkeren Strömen nach Aufhören der Hemmung sogleich ein gewöhnlicher Atmungstypus sich einstellt.

3) Während der Reizdauer seichte Atmung auch bei 22000 Ohm nach Hemmung; bei 5 and 6-7 MA. folgt während der Reizdauer der kompletten Hemmung eine seichte, etwas unregelmäßige Atmung resp. eine unvollständige Hemmung. Bei stärkeren Strömen wìrd die Wiederkehr der Atmung durch einige tiefere Atemzüge eingeleitet, nur bei $16 \mathrm{MA}$. die mäßig tiefere Atmung von längerer Dauer. 
G. Bikeles und L. Zbyszewski:

A. Kaninchen (Fortsetzung).

\begin{tabular}{|c|c|c|c|c|c|c|c|}
\hline \multirow{2}{*}{ 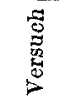 } & \multirow[b]{2}{*}{ ohm } & \multirow{2}{*}{$\begin{array}{c}\text { Milli- } \\
\text { ampère }\end{array}$} & \multirow{2}{*}{$\begin{array}{c}\text { Reiz- } \\
\text { dauer } \\
\text { Sek. }\end{array}$} & \multirow{2}{*}{$\begin{array}{l}\text { Verlang- } \\
\text { samung der } \\
\text { Atmung }\end{array}$} & \multicolumn{3}{|c|}{ Hemmung der Atmung } \\
\hline & & & & & anfängliche & \begin{tabular}{|c|} 
während der \\
Reizung
\end{tabular} & $\begin{array}{l}\text { Reizung } \\
\text { uiberdauernd }\end{array}$ \\
\hline \multirow[t]{3}{*}{$\left(V^{1}\right)$} & $22000)$ & ohne & 30 & $+\int_{\text {regel- }}^{\text {und un- }}$ & fast & & \\
\hline & $20000\}$ & Ausschlag & 30 & $\left\{\begin{array}{l}\text { regel- } \\
\text { mẳig }\end{array}\right.$ & gehemmt & & - \\
\hline & $\left.\begin{array}{l}18000 \\
18000\end{array}\right\}$ & $4-5$ & 30 & - & - & + & - \\
\hline \multirow{3}{*}{$\left.\nabla^{2}\right) !$} & $20000)$ & $\begin{array}{l}\text { ohne } \\
\text { Anssehlag }\end{array}$ & 30 & ) & & & \\
\hline & $17000\}$ & $3-4$ & 30 & 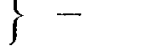 & - & - & - \\
\hline & 14000 & $6-7$ & 30 & 一 & + & - & - \\
\hline$\left.V I^{3}\right)$ & 18000 & $6-7$ & 30 & 一 & - & + & - \\
\hline
\end{tabular}

B. Hund.

\begin{tabular}{|c|c|c|c|c|c|c|c|}
\hline \multirow{2}{*}{ 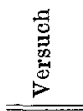 } & \multirow[b]{2}{*}{ Ohm } & \multirow{2}{*}{$\begin{array}{l}\text { Milli- } \\
\text { ampère }\end{array}$} & \multirow{2}{*}{$\begin{array}{l}\text { Reiz- } \\
\text { dnuer } \\
\text { Sek. }\end{array}$} & \multirow{2}{*}{$\begin{array}{c}\text { Verlang- } \\
\text { samung der } \\
\text { Atmung }\end{array}$} & \multicolumn{3}{|c|}{ Hemmung der Atmung } \\
\hline & & & & & anfängliche & $\mid \begin{array}{c}\text { während der } \\
\text { Reizung }\end{array}$ & $\begin{array}{c}\text { Reisung } \\
\text { überdauernd }\end{array}$ \\
\hline \multirow[t]{11}{*}{$\left.I^{4}\right)$} & 20000 & ohne Aussehl. & 30 & - & - & 一 & - \\
\hline & 17000 & $6-7$ & 30 & & & & \\
\hline & 15000 & 8 & 30 & & & & \\
\hline & 13000 & 8 & 30 & $T$ & - & - & - \\
\hline & 10000 & 9 & 30 & & & & \\
\hline & 7000 & 15 & 30 & & & & \\
\hline & 5000 & 20 & 30 & \multirow{2}{*}{\multicolumn{4}{|c|}{ Vergleiche anschließende Bemerkung. }} \\
\hline & 4000 & 25 & 30 & & & & \\
\hline & 3000 & 33 & 30 & & & & \\
\hline & 2000 & 46 & 30 & - & - & + & 一 \\
\hline & 1500 & 60 & 30 & - & - & - & + \\
\hline \multirow[t]{2}{*}{$\left.\operatorname{II} a^{5}\right)$} & 1200 & 65 & 25 & - & - & + & - \\
\hline & 1000 & 75 & 15 & - & - & - & + \\
\hline
\end{tabular}

1) Bei 4-5 MA. bei erster Reizung vor Schluß zwei Atemzüge, hingegen nach Aufhören der Reizung gerade، da Atmung unterbrochen, während die. selbe nach der nächsten Reizung (und derselben Stromstärke) sich regelmäBig abspielt.

2) Bei 20000 Ohm Verlangsamung nur in Beginn der Reizung; bei 3-4 MA. Atmung verlangsamt und seicht im Beginn und gegen Ende, bei 6-7 MA. nach Hemmung während der Reizung Atmung unregelmäßig, etwas verlangsamt und seicht, nachher Atmung ohne Störung.

3) Nach Aufhören der Reizung sogleich gewöhnliohe Atmung.

4) Von 6-7 bis 9 MA. Verstärkung der Atmung besonders im Beginn der der Reizung mit. einer Dauer parallel der Steigerung der Stromintensität. Von 15 bis 33 MA. nach anfänglicher Beschleunigung progressive Hemmung Nach Aufhören der Reizung (nicht konstant) Atmung vertieft.

5) ad a) Atmung nach Hemmung vertieft und beschleunigt. 
B. H und (Fortsetzung).

\begin{tabular}{|c|c|c|c|c|c|c|c|}
\hline \multirow{2}{*}{ 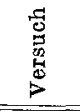 } & \multirow[b]{2}{*}{ Ohm } & \multirow{2}{*}{$\begin{array}{l}\text { Milli- } \\
\text { ampère }\end{array}$} & \multirow{2}{*}{$\begin{array}{c}\text { Reiz- } \\
\text { dazer } \\
\text { Sek. }\end{array}$} & \multirow{2}{*}{$\begin{array}{c}\text { Verlang- } \\
\text { samung der } \\
\text { Atmung }\end{array}$} & \multicolumn{3}{|c|}{ Hemmung der Atmung } \\
\hline & & & & & anfängliche & $\begin{array}{c}\text { während der } \\
\text { Reizung }\end{array}$ & $\begin{array}{l}\text { Reizung } \\
\text { iiberdauernd }\end{array}$ \\
\hline \multirow[t]{2}{*}{$\left.\operatorname{II} b^{1}\right)$} & \multicolumn{7}{|c|}{ nach Ausschaltung bei der Nn. vagi. } \\
\hline & $\begin{array}{r}1200 \\
\mathrm{~J} 000 \\
500\end{array}$ & $\begin{array}{c}73 \\
\text { über } \\
75\end{array}$ & 15 & - & - & - & - \\
\hline
\end{tabular}

Zu bemerken ist, $d a B$ die Atmungshe moung in allen obigen Versuchen entweder ungefähr in der Mitte zwischen Exund Inspirium oder ziemlich nahe der Kuppe des Exspiriums erfolgte. Manchmal hat es den Anschein, daß das Stadium der Atmung, in dem der Reiz einsetzte, dabei eine Rolle spiele, jedoch beim Gegeneinanderhalten einer größeren Versuchszahl ließ sich dafür gar keine Regel ausfindig machen.

Während wir bezüglich der im vorigen erörterten Erscheinungen seitens des Kreislaufs nicht zögern, dieselben einer Reizung bzw. Erschöpfung der entsprechenden Oblongatazentra zuzuschreiben, müssen wir uns bezüglich der Atmungshemmung Reserve auferlegen. In Anbetracht nämlich der Tatsache, daß die Reizung so mancher Hirnnerven eine Hemmung der Atmung herbeiführt, kann man - selbst bei direkter elektrischer Reizung der Oblongata - nie bestimmt wissen, ob die dabei beobachtete Hemmung die Folge einer Reizung des Zentrums oder nur. zentripetaler Bahnen sei. Wir wollen hier nur auf folgende Parallele zwischen dem Eintreten einer Atmungshemmung und dem Einsetzen des bereits mehrfach eirwähnten Extensionstonus bei Reizungen mittels Wechselströmen die Aufmerksamkeit lenken. He m mung der Atmung erfolgt beim Kaninchen schon bei Strö. men von sehr geringfügiger Intensität, und zwar zeigt sich teilweise (anfängliche) Hemmung schon bei einer so schwachen Stromstärke, bei welchem das Ampèremeter gar keinen Ausschlag gab; Hemmung wiederum während der ganzen Reizdauer erfolgt eventuell schon bei 2-5 MA. Bezüglich des Auftretens des Extensionstonus zeigte sich bei Kaninchen, bei denen mit sehr schwachen Strömen gereizt wurde, daß in der Regel ebenfalls schon geringfügige Stromintensitäten hinreichen, und $\mathrm{zwar}$ erfolgt bei 3-5 MA. ein ausge prägter Extensionstoñus; bei 20,000 Ohm (Ampèremeter ohne Ausschlag) zeigte sich eine merkliche Andeutung desselben.

1) ad b) Bei 73 MA. nach Hemmung während der Reizung Atmung sehr seicht und frequent, dieselbe bleibt frequent auch nach Aufhören der Reizung. Bei Strömen über $75 \mathrm{MA}$. ist dagegen nach Hemmung deutlich verlangsamt. 
Diese beiden Phänomene (von denen der Extersionstonus einer Reizung der Oblongata resp. eines entsprechenden Zentrums zuzuschreiben ist) gemeinschaftliche und ganz analoge überaus, Jeichte Auslösbarkeit schon bei minimalem Strome, wie das nicht seltene Vorkommen von Atmungshemmung nur im Beginne der Reizung, dürfte doch vielleicbt zugunsten der Annahme sprechen, daß es sich bei diesen Atmungshemmungen auch um Folgen einer Einwirkung auf das Atmungszentrum handle.

Anhang betreffend die Verwendung von Gleichstrom und dessen Einflu $B$ auf die Atmung.

Im Anschluß an die beschriebenen Versuche interessierte uns die Frage, ob tonisch-klonische Krämpfe auch bei Reizung mittels gleichgerichteter Ströme und zwar bei gleichartiger Applikation der Elektroden wie in den vorigen Versuchen eintreten. - Diese Versuche wurden nur an Kaninchen ausgeführt. Bei denselben wurde auch der Einfluß auf die Atmung beobachtet.

Als Stromquelle diente uns hier der durch einen rotierenden Transformator gelieferte, aus dem städtischen Wechselstrom ungewandelte Gleichstrom. Es zeigte sich nun, daß bei Anwendung desselben ein Extensionstonus sich hier und da präsentiert, aber keine irgendwie regelmäßige Erscheinung ist. So zeigte sich der Extensionstonus in zwei Versuchen erst bei $100 \mathrm{MA}$. In einem Versuch trat ein exquisiter Extensionstonus bei etwa 52 MA., bei einem anderen bei 60 MA. auf, während bei weiterer Steigerung der Stromintensität kein Extensionstonus mehr bei neuerlichen Reizungen zum Vorschein kam. Vereinzelte klonische Zuckungen an den Pfoten kamen bei Strömen von 15, 20, 30 MA. zum Vorschein; bei etwa $60 \mathrm{MA}$. (seltener bei $40 \mathrm{MA}$.) kam es zu anhaltenden durch die ganze Reizdauer sich wiederholenden klonischen Zuckungen, jedoch waren auch diese klonischen Krämpfe nicht konstant und dieselben fehlten evtl. sogar bei 100 MA. - Klonische Zuckungen an den Pfoten nach Aufhören der Reizung wurden bei Stromintensitäten von 80-100 MA. beobachtet.

Den Einfluß der Reizung mittels Gleichstroms auf die Atmung zeigt in den eirzelnen Versuchen die nachfolgende Zusammenstellung:

Versuch 1.

\begin{tabular}{|c|c|c|c|c|c|}
\hline \multirow[b]{2}{*}{ Milliampère } & \multirow[b]{2}{*}{$\begin{array}{l}\text { Reizdauer } \\
\text { Sek. }\end{array}$} & \multirow{2}{*}{$\begin{array}{l}\text { Verlang- } \\
\text { samung der } \\
\text { Atmung }\end{array}$} & \multicolumn{3}{|c|}{ Hemmung der Atmung } \\
\hline & & & anfängliche & $\begin{array}{l}\text { wäbrend der } \\
\text { Reizung }\end{array}$ & $\begin{array}{l}\text { Reizung } \\
\text { überdauernd }\end{array}$ \\
\hline 53 & 30 & - & $\cdots$ & + & - \\
\hline 5 & 30 & & & & \\
\hline 10 & 30 & + & - & - & - \\
\hline 15 & 20 . & 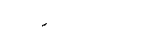 & & & \\
\hline 20 & 20 & + & - & - & - \\
\hline 25 & 20 & - & -+ & - & - \\
\hline 30 & 20 & & & & \\
\hline 40 & 20 & & & & \\
\hline 50 & 20 & & & & \\
\hline 60 & 20 & - & - & + & - \\
\hline 70 & 20 & & . & & \\
\hline 80 & 20 & & & & \\
\hline 90 & 20 & & & & \\
\hline 100 & 20 & - & $\ldots$ & - & $t$ \\
\hline 100 & 20 & - & & & \\
\hline
\end{tabular}


Bei 5-15 MA. Änderung der Atmung (unregelmäßig seicht) nur in Beginn. Bei $20 \mathrm{MA}$. hält seichte etwas verlangeamte Atmung während der ganzen Reizdauer an. Bei $25 \mathrm{MA}$. nach vollständiger Hemmung, während der weiteren Reizdauer abnehmend verlangsamte Atmung. Bei 30 und $33 \mathrm{MA}$. Hemmung während Reizdauer nicht ganz vollständig. Nach der Reizung bei 40-50 MA. einige tiefere Atemzüge; bei $60 \mathrm{MA}$. dauert etwas beschleunigte Atmung ziemlich lange an, dagegen bei 70-90 MA. nach Aufhören der Reizung Atmung alsbald von gewöhnlichem Typus.

Versuch 2.

\begin{tabular}{|c|c|c|c|c|c|c|c|}
\hline \multirow[b]{2}{*}{$\begin{array}{l}\text { Milli- } \\
\text { ampere }\end{array}$} & \multirow{2}{*}{$\begin{array}{l}\text { Reiz- } \\
\text { dauer } \\
\text { Sek. }\end{array}$} & \multirow[b]{2}{*}{ Anode } & \multirow[b]{2}{*}{ Kathode } & \multirow{2}{*}{$\begin{array}{l}\text { Verlang } \\
\text { samung der } \\
\text { Atmung }\end{array}$} & \multicolumn{3}{|c|}{ Hemmung der Atmung } \\
\hline & & & & & anfängliche & $\begin{array}{c}\text { während } \\
\text { der Reizung }\end{array}$ & $\begin{array}{c}\text { Reizung } \\
\text { überdauernd }\end{array}$ \\
\hline 50 & 20 & - & am Nacken & - & - & + & $\ldots$ \\
\hline 5 & 20 & - &, & & & & \\
\hline 5 & 20 & - & , & & & & \\
\hline 10 & 30 & -- & ," & & & & \\
\hline 10 & 30 & - & , & & & & \\
\hline 15 & 25 & - & , & + & - & - & - \\
\hline 15 & 30 & - & ," & & & & \\
\hline 20 & 25 & $\therefore$ & , & & & & \\
\hline 20 & 30 & - & 9 & & & & \\
\hline 30 & 30 & $\ldots$ & ,, & $\ldots$ & + & - & - \\
\hline 40 & 25 & $\ldots$ & , & & & & \\
\hline 40 & 20 & - &, & & & & \\
\hline 60 & 20 & - & , & - & - & + & - \\
\hline 70 & 30 & $\ldots$ & , & & & & \\
\hline 80 & 30 & - & " & & & & \\
\hline 90 & 30 & $\ldots$ & 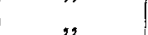 & & & & \\
\hline 100 & 20 & - & ," & - & - & - & + \\
\hline 100 & 30 & - & , & - & - & + & - \\
\hline 100 & 30 & am Nacken & - & - & - & - & t \\
\hline
\end{tabular}

Von 5-20 MA. gesehah die Einschaltung des Stromes abwechselnd plötzlich und langsam; im Resultat zeigte sich aber kein durebgreifender Unterschied. Nach der Reizung (und Hemmung) bei stärkeren Strömen: bei 60 MA. Atmung etwas beschleunigt, bei $90 \mathrm{MA}$. ist dieselbe lange unregelmäBig, dagegen bei $100 \mathrm{MA}$. Kathode am Nacken entweder vom normalen Typus oder eine Spur beschleunigt; bei 100 MA. Anode am Nacken, nach Hemmung verlangsamte, unregelmäßige und lange anhaltende Atmung.

Versuch 3.

\begin{tabular}{|c|c|c|c|c|c|}
\hline \multirow[b]{2}{*}{ Milliampère } & \multirow[b]{2}{*}{$\begin{array}{c}\text { Reizdauer } \\
\text { Sek. }\end{array}$} & \multirow[b]{2}{*}{ Anode } & \multirow[b]{2}{*}{ Kathode } & \multicolumn{2}{|c|}{ Hemmung der Atmung } \\
\hline & & & & $\begin{array}{l}\text { während der } \\
\text { Reizung }\end{array}$ & $\begin{array}{c}\text { Reizung } \\
\text { überdauernd }\end{array}$ \\
\hline 40 & 20 & - & am Nacken & & \\
\hline 50 & 20 & - & 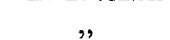 & & \\
\hline 60 & 20 & - & , & & \\
\hline 70 & 20 & - & 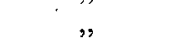 & + & -- \\
\hline 80 & 20 & - & $"$ & & \\
\hline 90 & 20 & - & 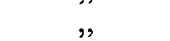 & & \\
\hline 100 & 20 & - & $"$ & & \\
\hline 100 & 20 & - & $"$ & - & + \\
\hline
\end{tabular}


Versuch 3 (Fortsetzung).

\begin{tabular}{|c|c|c|c|c|c|}
\hline \multirow[b]{2}{*}{ Milliampère } & \multirow[b]{2}{*}{$\begin{array}{c}\text { Reizdauer } \\
\text { Sek. }\end{array}$} & \multirow[b]{2}{*}{ Anode } & \multirow[b]{2}{*}{ Kathode } & \multicolumn{2}{|c|}{ Hemmung der Atmung } \\
\hline & & & & $\begin{array}{l}\text { während der } \\
\text { Reizung }\end{array}$ & $\begin{array}{c}\text { Reizung } \\
\text { uberdauernd }\end{array}$ \\
\hline 40 & 20 & am Nacken & - & & \\
\hline 50 & 20 &, & - & t & - \\
\hline 60 & 20 &, & - & & \\
\hline 70 & 20 & 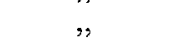 & - & & \\
\hline 80 & 20 &, & - & 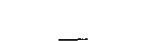 & \\
\hline 90 & 20 &, & $\ldots$ & 一 & + \\
\hline 100 & 20 & $\because$ & $\ldots$ & & \\
\hline 100 & 20 & $"$ & - & & \\
\hline
\end{tabular}

Bei Reizungen von 40-100 MA. (Kathode am Nacken) ist die Atmung nach Aufhören der Reizung und Hemmung fast ohne Störung, nur die ersten (ein bis drei) Atemzüge sind manchmal etwas unregelmäßig evtl. etwas seichter.

Bei Reizungen 40 und $50 \mathrm{MA}$. (Anode am Nacken) ist die wiederkehrende Atmung ohne jede deutliche Störung.

Hemmung bei $70 \mathrm{MA}$. (Anode am Nacken) dauerte länger an als bei 80 und $90 \mathrm{MA}$, und beim Wiedereinsetzen der Atmung war dieselbe gerade bei $70 \mathrm{MA}$. verlangsamt. und seicht.

Nach erster Reizung bei einer Intensität von $100 \mathrm{MA}$. (Anode am Nacken) ist die spontane wiedereinsetzende Atmung zunächst ohne Störung, wird aber nach Ablauf von mehreren Sekunden zunehmend langsamer und seichter, so daß man zur künstlichen Atmung schreiten mußte.

Versueh 4.

\begin{tabular}{|c|c|c|c|c|c|c|c|}
\hline \multirow[b]{2}{*}{$\begin{array}{c}\text { Milli- } \\
\text { ampère }\end{array}$} & \multirow{2}{*}{$\begin{array}{c}\text { Reiz- } \\
\text { dauer } \\
\text { Sek. }\end{array}$} & \multirow[b]{2}{*}{ Anode } & \multirow[b]{2}{*}{ Kathode } & \multirow[b]{2}{*}{$\begin{array}{l}\text { Verstärkung } \\
\text { der Atmung }\end{array}$} & \multicolumn{3}{|c|}{ Hemmung der Atmung } \\
\hline & & & & & anfängliche & $\left|\begin{array}{c}\text { während } \\
\text { der Reizung }\end{array}\right|$ & $\begin{array}{l}\text { Reizung } \\
\text { iiberdauernd }\end{array}$ \\
\hline 5 & 20 & - & am Nacken & & & 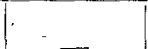 & ’ \\
\hline 5 & 20 & am Nacken & - & & - & - & \\
\hline 10 & 20 & - & am Nacken & & & & \\
\hline 10 & 20 & am Naoken & - & -1 & 一 & $\ldots$ & - \\
\hline 15 & 20 & - & am Nacken & + & & & - \\
\hline 15 & 20 & am Nacken & - & & & & \\
\hline 20 & 20 & - & am Nacken & - & + & - & $\ldots$ \\
\hline 25 & 20 & - & " & & $T$ & & - \\
\hline 30 & 20 & - & $"$ & & & & \\
\hline 20 & 20 & am Nacken & - & - & - & nicht & - \\
\hline 25 & 20 & $"$ & - & & & nicht voll- & \\
\hline 30 & 20 & , & - & & & & \\
\hline 40 & 20 & - & am Nacken & & & & \\
\hline 40 & 20 & am Nacken & - & - & - & + & - \\
\hline 50 & 20 & - & am Nacken & & & & \\
\hline 50 & 20 & am Nacken & - & & & & \\
\hline 60 & 20 & - & am Nacken & - & - & - & - \\
\hline
\end{tabular}


Versuch 4 (Fortsetzung).

\begin{tabular}{|c|c|c|c|c|c|c|c|}
\hline \multirow[b]{2}{*}{$\begin{array}{l}\text { Mrilli- } \\
\text { ampère }\end{array}$} & \multirow{2}{*}{$\begin{array}{c}\text { Reiz- } \\
\text { daner } \\
\text { Sek. }\end{array}$} & \multirow[b]{2}{*}{ Anode } & \multirow[b]{2}{*}{ Kathode } & \multirow[b]{2}{*}{$\begin{array}{l}\text { Verstärkung } \\
\text { der Atmung } \\
\text { l }\end{array}$} & \multicolumn{3}{|c|}{ Hemmung der Atmung } \\
\hline & & & & & anfängliche & $\begin{array}{c}\text { während } \\
\text { der Reizung }\end{array}$ & $\begin{array}{c}\text { Reizung } \\
\text { iuberdauerno }\end{array}$ \\
\hline 60 & 20 & 一 & am Nacken & - & - & - & + \\
\hline 60 & 20 & am Nacken & $\ldots$ & & & & \\
\hline 70 & 20 & - & am Nacken & & & & \\
\hline 70 & 20 & am Nacken & - & & & & \\
\hline 80 & 20 & - & am Nacken & - & - & + & - \\
\hline 80 & 20 & am Nacken & - & & & & \\
\hline 100 & 20 & $\ldots$ & amNacken & & & & \\
\hline 100 & 20 & - & $"$ & & . & & \\
\hline 100 & 20 & am Nacken & 一 & & & & \\
\hline $\left.100^{1}\right)$ & 20 &, & - & . & - & 一 & + \\
\hline
\end{tabular}

Bei 10 und 15 MA. ist Atmung im Beginn der Reizung beschleunigt und tiefer und ausgesprochener bei der Intensität von $10 \mathrm{MA}$. als bei $15 \mathrm{MA}$. Bei Anode am Nacken nach dieser anfänglichen Verstärłung der Atmung wird dieselbe anscheinend verlangsamt bis zum Schluß der Reizung. Bei Stromintensitäten bis 50 MA. ist die nach der Hemmung wiedereinsetzende Atmung von gewöhnlichem Typus. Bei $60 \mathrm{MA}$. (sowohl bei Kathode wie bei Anode am Nacken) ist die Atmung nach Ablauf der vollständigen Hemmung deutlich verlangsamt, dagegen bei 70, 80, 100 MA. und Kathode am Nacken ist wiederauftretende Atmung ohne Störung; bei Anode am Nacken ist dieselbe bei 70-80 MA. etwas seichter evtl. verlangsamt und die Verlangsamung ist am deutlichsten bei $100 \mathrm{MA}$. (Anode am Halsteil).

Beachtenswert ist, daB die Verlangsamung bei 60 MA. bezüglich bei 70 bis $80 \mathrm{MA}$. nicht die ersten zwei bis drei Atemzüge, sondern die nachfolgenden betrifft.

Es sei nur darauf hingewiesen, daß bei diesen Reizungen mittels Gleichstromes unvergleichlich viel stärkere Ströme zur Erzeugung einer Hemmung nötig sind als bei Anwendung von Wechselströmen.

Die Hemmung exfolgt auch da (regallos), sei es ungefähr in der Mitte zwischen Ex- und Inspirium oder ziemlich nahe der Kuppe vom Exspirium; nur bei zwei Reizungen - denen eine seichte Atmung voranging - zeigt die Kurve eine Hernmung näher der Kuppe vom Inspirium.

\section{Zusammenfassung der Ergebnisse:}

Der Effekt der Hirn-(Oblongata-)Reizung mittels Wechselströmen bewährte sich in diesen Versuchen, bei Berücksichtigung individueller Verhältnisse als eine konsequente.

Bezüglich des Vaguszentrums ist die sehr häufige, den gewöhnlichen Vaguspulsen vorausgehende, gänzliche Hernmung der Herztätigkeit, die aber nur sehr kurz anhält, hervorzuheben. Diese anfängliche kurzdauernde Hemmung der Herztätigkeit ist in Parallele zu bringen mit dem ebenfalls am Anfang der Reizung auftretenden und bald

1) Anode ausnahmsweise am Halsteil (d. i. tiefer unten als sonst). 
im Laufe einer längeren Reizung verschwindenden, exquisiten Tonus der Körpermuskulatur.

Nach Einstellung der Reizung erfolgte bei manchen Versuchstieren eine vorübergehende, auffallende Beschleunigung der Herztätigkeit, also eine zeitweilige Lähmung (Erschöpfung) des Vaguszentrums.

Bezüglich des vasomotorischen Zentrums zeigte sich bei manchen Versuchstieren unter dem Einfluß der angewandten Reizungen eine derartige Erschöpfung des vasomotorischen Oblongatazentrums, daß nach vorübergehender Reizung nach Einstellung derselben der Blutdruck vorübergehend so sehr sank, wie nach faktischer Ausschaltung der Oblongata, d. h. der restierende Blutdruck entspricht einem vasomotorischen Tonus der Rückenmarkszentren.

Bezüglich der Atmung ist schwer zu entscheiden, ob die festgestellte Hemmung der Ausdruck einer Reizung des Zentrums oder zentripetaler Bahnen ist. Es muß aber auf einen evtl. anfänglichen Charakter der Hemmung bei Verwendung schwacher Ströme wie auf die leichte Auslösbarkeit der Hemmung überhaupt (analog zur leichten Auslösbarkeit des Extesionstonus) hingewiesen werden.

Im Gegensatz zu den Wechselströmen zeigt sich der Effekt der Reizung mittels Gleichstroms als ein sehr geringer und sehr wechselnder. 